\title{
Criterios personalistas para una evaluación antropológica del empleo de las tecnologías en la escuela
}

\author{
Vera González, Raquel \\ Revista Educación, vol. 44, núm. 1, 2020 \\ Universidad de Costa Rica, Costa Rica \\ Disponible en: http://www.redalyc.org/articulo.oa?id=44060092016 \\ DOI: https://doi.org/10.15517/revedu.v44i1.35406
}

Criterios personalistas para una evaluación antropológica del empleo de las tecnologías en la escuela

Esta obra está bajo una Licencia Creative Commons Atribución-NoComercial-SinDerivar 3.0 Internacional. 
Artículo científico

\title{
Criterios personalistas para una evaluación antropológica del empleo de las tecnologías en la escuela
}

\author{
Raquel Vera González \\ Universidad a Distancia de Madrid, España \\ masterantropologia@personalismo.org \\ iD http://orcid.org/0000-0002-1299-4474
}

Personalist Criteria for an Anthropological Assessment of Technology Use at School

DOI: https://doi.org/10.15517/revedu.v44i1.35406

Redalyc: http://www.redalyc.org/articulo.oa?id=44060092016

Recepción: 31 Enero 2019

Aprobación: 04 Octubre 2019

\section{Resumen:}

Este ensayo ofrece una lectura personalizante del uso de las Tecnologías de la Información y la Comunicación (TIC) en la escuela, con el objetivo de mostrar la importancia del personal docente como educador que interpela los diferentes dinamismos antropológicos del educando: el intelectivo, como también el volitivo y afectivo. Cada docente interpela estos dinamismos de manera distinta a las TIC; la motivación que genera en el educando no es equiparable a la de la concepción del profesorado principalmente como facilitador de información. Así, a través de una revisión crítica de estudios relevantes en torno al uso de las tecnologías, y desde el enfoque de autores cercanos al personalismo integral, se presenta la necesidad antropológica de mantener el encuentro entre el cuerpo docente y el educando para contribuir adecuadamente al desarrollo personal del educando. Se concluye que un encuentro que puede ser sostenido y reforzado por las TIC como herramienta de apoyo puntual, no ha de ser suplantado por las TIC como principal instrumento de trabajo cuando este sustituya la relación interpersonal. Queda justificada la pertinencia de un sistema educativo que, o bien renuncia al empleo de las TIC en favor de habilidades distintas a las técnicas, o bien las introduce de manera revisable, crítica y limitada, teniendo en cuenta: lo obsoleto e intuitivo del aprendizaje del lenguaje tecnológico, y la contingencia humana para la adquisición de otras habilidades no tecnológicas.

Palabras ClaVE: Educación, Encuentro, Docente, Personalismo, TIC.

\section{Abstract:}

This study provides a personalized interpretation on the use of Information and Communication Technologies (ICT) at schools with the objective of demonstrating the importance of teachers as educators, particularly with regards to questioning anthropological dynamics of learners which include intellectual, volitional and emotional aspects. Teachers have a different approach towards these dynamics than do ICTs and teacher motivation is not comparable to that of being perceived as a mere facilitator of information. Thus, through a critical review of relevant studies regarding use of technology at schools by authors with a perspective of comprehensive personalism, it is argued that there is an anthropological need for a teacher-student encounter to appropriately contribute to the personal development of the learner. It is concluded that this encounter may be sustained and reinforced by ITCs as a specific support mechanism and that this interpersonal relationship cannot be replaced by ICTs. Thus, educational systems must either relinquish the use of ICTs in favor of other non-technical skills or use ICTs in such a manner where its use can be reviewed, assessed and limited without losing sight of the obsolete and intuitive nature associated with learning through technology and highlighting the importance for human beings to acquire other skills unrelated to technology.

KEYWORDS: Education, Encounter, Teachers, Personalism, ICT.

\section{INTRODUCCIÓN}

La sociedad actual habita principalmente en un mundo globalizado mediado por las tecnologías. La persona inmersa en el mundo de hoy crece dentro de esta realidad, hasta el punto de hablarse de ella en términos de ciborg humano (Szalay, 2016). Esto ha supuesto una serie de avances loables en ámbitos como la medicina, la economía, las comunicaciones, etcétera. Sin embargo, cada vez más estudios concluyen también en contra de lo que la inteligencia artificial está suponiendo para el crecimiento de lo más personal en el ser humano. 
Entre estos estudios, nos centraremos en aquellos que traten acerca de consecuencias de la exposición a las pantallas que afectan a la motivación interna, la atención, o las relaciones interpersonales en la población en edad escolar.

Este ensayo tiene como objetivo presentar en qué consiste el proceso personalizante que supone la educación formal en el ámbito escolar desde la perspectiva del personalismo integral ${ }^{[1]}$. Para ello, se presentan aquellos aspectos según los cuales la tecnología no puede plegarse a un criterio personalista sin menoscabo del crecimiento de la persona en sus dinamismos antropológicos; promoviendo el crecimiento humano en aquello que es específico de la persona, y desde donde puede seguir usando las tecnologías para su propio bien, sin quedar, en parte, anulado por las mismas en lo referente a aquellos dinamismos.

En el primer apartado, se cuestionará el alcance de tres informes de referencia internacional sobre la repercusión de la exposición a las pantallas en el desarrollo de la población escolarizada.

Del segundo al quinto apartado, se aborda la perspectiva antropológica del personalismo integral acerca de la figura del y la docente, y su relación con el educando. Para ello, se acude a las obras indicadas en la bibliografía de los autores Stein (1998), Burgos (2003, 2012, 2015 y 2019), Guardini (2002), Scheler (1998 y 2001), Von Hildebrand (1983, 1996, 1997 y 1998), Wojtyla (2009 y 2011), Allen (2014) y Crosby (2007).

En el apartado sexto, se exponen las ventajas e inconvenientes que pueden ofrecer las TIC en relación a la importancia de la relación educador-educando fundamentada en los anteriores apartados. En el séptimo apartado, se discuten los aspectos abordados en el sexto apartado desde la comprensión del personalismo integral acerca del proceso personalizante que realiza al ser humano en aquello que le es más propio, aquello que más le humaniza en los términos descritos en los apartados del segundo al quinto.

En el octavo apartado, se presenta una propuesta para el uso de las TIC en base a la teoría del conocimiento de Burgos (2015) por proporcionar esta una visión personalista integradora de las vivencias humanas y, de este modo, de toda la persona. En el noveno apartado, se consideran las razones relevantes indicadas por L'Écuyer (2015) que han dado lugar a la implantación de las TIC en el aula; y se evalúan, principalmente, en función de la concepción que el personalismo integral ofrece del ser humano. El ensayo termina con las conclusiones.

\section{EVIDENCIAS ACERCA DEL IMPACTO DE LAS TIC EN EL RENDIMIENTO ACADÉMICO Y EN EL PROCESO DE REALIZACIÓN DE LA PERSONA}

Resultaría excesivo recoger aquí todos los estudios sobre el tema, pero conviene mencionar tres de ellos, y algunas de sus afirmaciones, que se señalan a continuación ${ }^{[2]}$. Se han escogido estos informes por ser referentes a nivel internacional y, especialmente, para la cultura occidental; por recoger el resultado de un importante número de estudios sobre la cuestión; y por pronunciarse sobre puntos clave en torno a los cuales se va a analizar la cuestión.

- Fuchs y Wössmann (2005), en un análisis amplio de los resultados PISA y otros 29 estudios, anuncian que el uso de dispositivos electrónicos en los colegios no parece contribuir sustancialmente al aprendizaje de las habilidades básicas como matemáticas o lengua. Indica, sin embargo, que un uso comedido del ordenador y de internet en el colegio, mejora el rendimiento académico, mientras que, si se usa varias veces a la semana, lo empeora. Y remarca que, dicho empleo, puede empeorar los resultados en habilidades tan básicas como las matemáticas y la escritura:

Having a computer at home and using it at school will almost certainly raise some computer skills. What our results suggest is only that this may come at the expense of other skills. However, the results in Borghans and ter Weel (2004) show that these other (math and writing) skills are the ones that yield significant labor-market returns, not the computer skills. (p. 18)

- Sobre la base de 22 estudios, la Organización para la Cooperación y el Desarrollo Económicos (OCDE, 2015) concluye de modo similar a Fuchs y Wössmann (2005), afirmando en un informe relativo a 64 países, 30 de los cuales no pertenecen a la OCDE: 
...in countries where it is more common for students to use the Internet at school for schoolwork, student's performance in reading declined between 2000 and 2012, on average. (...) In fact, PISA results show no appreciable improvements in students achievement in reading, mathematics or science in the countries that have invested heavily in ICT for education.... while PISA results suggest that limited use of computers at school may be better than not using computers at all, using them more intensively than the current OECD average tends to be associated with significantly poorer student performance. (pp. 15-16)

- Sobre la base de 49 estudios, la Academia Americana de Pediatría (AAP, 2016) recomienda evitar el uso de cualquier medio digital a menores de 2 años; hasta los 5 años, lo limita a un máximo de una hora al día si así lo desean los padres. Después de los 6 años, pide que los padres se aseguren de que el uso no interfiera con el sueño, la actividad física, el tiempo de relación en familia y con los amigos, actividades en el exterior, así como que las habitaciones estén libres de este tipo de medios.

El uso de las tecnologías, por tanto, no impacta solo positivamente en la adquisición de habilidades técnicas, sino que también puede impactar negativamente en la integración de la persona y su realización. El informe de la AAP (2016) habla de afectación del sueño, percepción de la realidad offline, tiempo de encuentro interpersonal, y de la necesidad de intimidad e interioridad en las habitaciones libres de dispositivos electrónicos. Queda así indicado que no se puede implantar las TIC de manera indiscriminada, y que, si las habitaciones deben estar libres de este tipo de medios, los padres han de poner límites al uso de las TIC, pero ¿solo en las habitaciones?

La exposición a dispositivos electrónicos afecta a la manera de enfrentarse a la realidad: si tenemos en cuenta que, como apunta la OCDE, el tiempo de exposición a la pantalla en la escuela afecta al rendimiento académico, y, como apunta la AAP (2016), también incide en los factores de riesgo señalados como la actividad física, el sueño o las relaciones interpersonales. En un discurso personalista, la persona no separa su percepción de la realidad aprendida en la escuela virtualmente, de lo vivenciado fuera del uso de los dispositivos electrónicos; sino que trata de unificar sus vivencias, creando estas en ella una serie de tendencias, hábitos, disposiciones, afectación, no solo habilidades. El personalismo integral apunta hacia la concepción de la persona y de su desarrollo desde la integración de la persona y sus experiencias (Burgos, 2003 y 2015; Guardini, 2002; Stein, 1998), por lo que conviene evaluar qué experiencias le humanizan y cuáles requieren un límite definido para no contribuir a su despersonalización.

¿Por qué esa diferenciación en la edad por parte de la Academia Americana de Pediatría? Como explicita el citado informe de la AAP (2016) y explica el neurocientífico Spitzer (2013), el desarrollo del niño y de la niña, de su personalidad, de su cerebro, están en juego, pero éfinaliza a los 6 años de manera que ya no sería necesaria una pauta clara en el ámbito escolar? Desde la filosofía no se pueden proporcionar pautas pedagógicas sin interdisciplinariedad. Sin embargo, sí se pueden aportar criterios basados en la antropología para valorar la pertinencia o no de determinadas acciones y métodos pedagógicos interpretando datos sociológicos, extrayendo conclusiones relativas a la estructura de la persona. El personalismo integral entiende a la persona desde sus dinamismos, y es desde estos que puede considerar más o menos adecuadas unas medidas pedagógicas por presentarse más o menos acordes al desarrollo de los dinamismos propios de la persona humana. Se presentan a continuación algunos de estos criterios e interpretaciones en base al pensamiento de los autores personalistas indicados en la introducción. Los criterios de los cuatro siguientes apartados permitirán evaluar la pertinencia del empleo de las TIC en la escuela.

\section{LA TAREA EDUCATIVA: OBJETIVOS Y EXPERIENCIA DE LA PROPIA CONTINGENCIA}

En un pequeño inciso sobre la relación entre la condición humana y la educación, la filósofa y educadora personalista Stein (1998) alude a la importancia de dos objetivos pedagógicos: 1) facilitar el acceso a los bienes espirituales objetivos (no solo a los conocimientos, sino también a la experiencia y al testimonio de una vida lograda), y 2) la capacitación para la autodeterminación, para decidir libremente entre las múltiples 
posibilidades, mediante una dirección y seguimiento. ¿Sería necesaria dicha dirección si el ser humano no tuviese límite ni ontológico ni cronológico?

Sin tener en cuenta la propia contingencia, la educación puede implantar sin mucho problema un sistema educativo basado en la multitarea, por ejemplo. Asimismo, podría fomentarse una educación completamente autodidacta, y sin importar si se desarrollan más unas capacidades que otras, porque: o se considera que todas se pueden desarrollar a la vez, o se considera que se podrán desarrollar a posteriori, a voluntad del educando. Es el modelo del ensayo-error absolutizado, del alumnado autodidacta ante un facilitador de medios o de información el cual no precisa dirigir su voluntad ni con su ejemplo, ni conduciendo al alumno a ejecutar actos que afiancen unos hábitos y habilidades por encima de otros. Este modelo encaja con una comprensión del hombre cuya libertad está exenta de objetivo, absoluta en términos nietzscheanos. En esta comprensión, el hombre crea sus propios valores por medio de sus actos; se crea a sí mismo, en términos sartreanos.

Sin embargo, siguiendo a Stein (1998) en su descripción fenomenológica de la fuerza anímica del ser humano:

Al hombre no le es posible desarrollar todas sus potencias simultáneamente y en igual medida, al igual que tampoco puede actualizarlas todas a la vez (...) El alma parece disponer de una cantidad concreta de fuerza, que puede ciertamente ser empleada en diversas direcciones, pero con la limitación de que su empleo en una de ellas priva de fuerza a las direcciones restantes. (p. 138).

Esta descripción, aplicada al uso de las TIC, queda avalada por estudios como los desarrollados por Ophir, Nass y Wagner (2009), Madigan et al. (2019), o Spitzer (2013). Esto es, si partimos del dato antropológico de la contingencia del ser humano: emplear tiempo y fuerzas en desarrollar habilidades más técnicas implica una disminución en la dedicación al desarrollo de otras habilidades.

\section{LA TAREA EDUCATIVA, EL AUTODOMINIO, EL ENCUENTRO Y LA CONCIENCIA DE Sí MISMO}

A pesar de referirse a la contingencia de la persona como elemento a tener en cuenta en la educación, Stein (1998) -al igual que otros autores personalistas como Von Hildebrand (1983) o Burgos (2003)-, destaca la libertad y la espiritualidad como lo específico del ser humano, y entiende esta libertad como autodominio que se va adquiriendo. El hombre, ni nace con el desarrollo pleno de esta capacidad, ni se le puede exigir en igual grado a un infante ante la estimulación de una pantalla que a un adulto, o incluso que a un joven universitario. Es más, se ha de velar especialmente por el desarrollo del autodominio, por cuanto que "toda decisión crea una disposición a volver a tomar otra decisión análoga" (Stein, 1998, p. 150) que contribuye o no a mi vivir desde mi de tal modo que pueda decidir desde mí mismo. En otros términos, se denomina también forja del carácter. La interioridad de ese vivir desde sí mismo, más allá de impulsos internos o externos, se muestra clave para desarrollar ese autodominio desde la autoconciencia.

Para llegar a esa interioridad, he de encontrarme con un tú ${ }^{[3]}$ que me haga consciente de la diferencia de mi yo; que me lleve a replegarme en $m i$ interioridad para, creciendo desde ella, trascenderme de nuevo a ese tú. Un tú al que poder volver a enriquecer desde un proyecto común que compartir; un tú que responda de la realidad relacional del ser humano, de su necesidad promocional mediante el compromiso, y no solo mediante el respeto distante. Es importante el encuentro interpersonal, y es necesario el tiempo para darse cuenta del sí mismo. Y este encuentro, cuando se da entre el educando y el profesorado, ha de permitir una relación jerárquica que es la que muestra al educando que se puede crecer hacia la propia realización, sin estancarse en una relación horizontal. En palabras del pensador personalista Scheler (2001):

El cambio de la disposición de ánimo es un proceso moral al que no puede determinar nunca la orden (ni aun siquiera la orden de uno mismo, caso de que existiera), ni menos la instrucción pedagógica (que no alcanza a la disposición de ánimo), ni tampoco el consejo y el asesoramiento, sino únicamente el seguir al prototipo. (p. 741) 


\section{LA TAREA EDUCATIVA Y LA AFECTIVIDAD}

Aunque Stein (1998) no sitúa explícitamente la afectividad entre los objetivos de la educación en el marco de la condición humana, estos están implícitos al mencionar la necesidad de dar dirección y seguimiento a la formación de la voluntad. La figura del y la docente, en cierta medida aparece, así como el apego que proporciona seguridad y confianza en sí mismo en esta interrelación con aquel que asegura ya al educando, desde una vida más lograda, que es posible alcanzarla, y que se está capacitando para ello.

Una educación enfocada a la mera transmisión de conocimientos por medios cada vez más tecnológicos y tecnocráticamente enfocados, o, incluso, una educación enfocada a la repetición de actos, sin tener en cuenta la motivación interna del educando y su afectividad; se tornaría, a la larga, ineficaz, insulsa, desintegradora de la persona (Feinstein, 2006; L'Écuyer, 2015). Ahora bien, en el personalismo integral, la persona queda asumida como una unidad; bajo esta lectura, una educación tal no lograría hacer desaparecer la empatía humana requerida para el encuentro entre el profesorado y el educando, pero la relegaría a un comportamiento por motivación externa más que interna, en lugar de ser encauzada desde una afectividad espiritual que, en términos de Von Hildebrand $(1996,1997)$, conociese la causa de sus afectos y efectuase una lectura personalista de estos. En una comprensión pedagógica tal, casa docente funciona más como medio que como fin en sí, y transmite, por ese mismo comportamiento, una comprensión del educando desde la gramática del funcionamiento y de la eficiencia.

$\mathrm{Si}$, por el contrario, se procura educar el corazón para que sepa descubrir y secundar un valor inteligible e inigualable en sí mismo, en el otro, en el mundo offline que le rodea, entonces el alumnado podrá situar el valor de lo que representa el mundo online en su justa medida, y comprometerse con él (L'Écuyer, 2015). Pero dicha valoración requiere un tiempo de encuentro que no puede ser sistemáticamente sustituido por el traslado de información a otro por medio de una vía telemática. La vía telemática puede secundar este encuentro, pero no interferir en él si no se considera necesario ${ }^{[4]}$. La cita de Scheler (2001), enunciada en el anterior apartado de este ensayo, proporciona la clave: el especial encuentro interpersonal que tiene lugar entre el educando y el profesorado, podrá mover la voluntad hacia el bien de las personas implicadas, así como abrir a la persona mediante una implicación personal que le permita entender mejor los contenidos que se le ofrecen en el ámbito educativo, si no se limita a convertir al educador en un mero facilitador/a de información o coordinador/a de aula tecnológica.

Siguiendo a Von Hildebrand (1983), la afectividad espiritual es aquella que responde al valor positivo, a lo que importa; pero la voluntad se mueve hacia aquello a lo que da importancia, y se da importancia a aquello a lo cual se dedica tiempo. Por lo que la voluntad puede dar importancia a algo que alimente una afectividad no espiritual o menos espiritual ${ }^{[5]}$. Si se sitúa al alumno o alumna 4 horas diarias ante una pantalla que ofrece unos estímulos irreproducibles por el mundo offline, por parte del facilitador/a de información o docente; el docente deja de tener importancia en etapas en las que el autocontrol, la autoconciencia y la capacidad de trascendencia se desarrollan o no de manera especial mediante los propios actos y vivencias, dando lugar a la propia personalidad. Esta relación entre el profesorado y el educando deja de tener todo el potencial educativo del encuentro, un potencial llamado a sacar lo mejor del educando y no solo a lidiar con las circunstancias digitalizadas del mismo.

El educando está llamado a integrar tanto la autoconciencia, como la autotrascendencia, el autodominio o la dirección de los afectos hacia lo que importa porque personaliza, permite descubrir la irrepetibilidad del otro y de sí mismo, y vivir desde ella en la interioridad. Solo desde la interioridad, e integrando todas las dimensiones de la persona, incluida la afectividad, hacia lo que importa en la labor de llegar a ser persona; la persona se puede entregar de manera no objetualizante de sí misma o de otro, es posible el encuentro y el compromiso (Crosby, 2007). Elementos ambos necesarios para la maduración de la persona en la asunción de responsabilidades, en su capacitación para responder de, para ser y saberse autor, y, de esa manera, aprender de los propios errores, porque pueden ser considerados como propios. Descubriendo en esta autoría mi ser como 
irrepetible, de índole personal. Mi interacción con el mundo real, en especial con el mundo de las relaciones interpersonales, en la medida en que estas responden de mi realidad relacional y de mi destino, no se pueden deshacer o hacer desaparecer como los contenidos de una web o aplicación; ni sustituir, porque me ayudan a crecer en esa autoría de los propios actos y en una asunción auténtica de la responsabilidad interpersonal (Wojtyla, 2011).

\section{LA TAREA EDUCATIVA Y LA UNIDAD PSICO-SOMÁTICA-ESPIRITUAL DE LA PERSONA}

En línea con la dimensión tripartita y los tres dinamismos desde los que se desarrolla la estructura personal en Burgos (2003) ${ }^{[6]}$; el ser humano precisa para su crecimiento personalizador de una atención también al desarrollo del propio cuerpo como lugar de manifestación de la persona, que participa de la dignidad de la misma. Sin embargo, una exposición demasiado prolongada a la pantalla, si bien mantiene a los niños y niñas inmersos en la misma en una primera etapa, más controlables a corto plazo por los profesores, también genera problemas físicos en un porcentaje significativo del alumnado[7], problemas que obstaculizan la disposición del cuerpo para las acciones de la voluntad que conducen al encuentro.

Así, Spitzer (2013) relaciona el uso de los medios digitales con insomnio, depresión, adicción, entre otros muchos males a los que considera innecesario exponer a los niños y niñas. Según el neurocientífico, estas consecuencias tienen más tiempo en ellos para enquistarse, dado que carecen todavía del autodominio necesario para hacer frente a la estimulación digital que no se puede reproducir en el mundo real del mismo modo.

Los estudios de Twenge, Joiner, Rogers y Martin (2018) y de Twenge, Martin y Campbell (2018) dirigidos a adolescentes, respecto al uso de ciertos dispositivos electrónicos, apuntan hacia consecuencias similares: infelicidad, suicidio, inmadurez. $\mathrm{Al}$ adolescente no le da tiempo a vivir desde la importancia del mundo real de las relaciones que pueden dar respuesta adecuada a su dignidad por medio del qué bueno que tú existas del amor interpersonal que se produce en el encuentro. Un encuentro que se produce en el tiempo que damos al otro para que se introduzca en la propia conciencia formando parte de un proyecto común, un nosotros que nos trasciende e interpela, que personaliza en una medida cualitativamente superior a la atención a los dispositivos electrónicos, y a lo que ofrece dicha atención. Las TIC tienen que encontrar su sitio como posibilitadoras de este encuentro, pero no como sustitutas, si queremos que contribuyan a un proceso de personalización. El infante, y el adolescente, aunque en otros parámetros, están creciendo y aprendiendo a discernir entre su imaginación y el mundo real, el mundo ideal y el concreto. La exposición continuada a un mundo virtual que me interpela desde fuera, corre el riesgo de desplazar la importancia del mundo real, y hacer que el mismo mundo virtual pierda significación personalizadora, generando incluso trastornos como el denominado autismo virtual ${ }^{[8]}$ (Oestreicher, 2013).

\section{LAS BONDADES DE LAS TECNOLOGÍAS EN EL AULA}

Sin olvidar que el potenciamiento de unas habilidades y facultades actúa en detrimento de otras por la limitación espacio-temporal del ser humano (Stein, 1998), los estudios al principio referidos afirman que un uso comedido de las tecnologías parece mejorar el rendimiento escolar. Pero ¿en qué puede consistir dicho uso comedido si se expone tanto a infantes como a adolescentes a unas consecuencias a largo plazo devastadoras según los estudios expuestos? ¿Por qué las estadísticas de Enkvist (2012) [9] al valorar diferentes sistemas educativos, no reflejan la necesidad de tal exposición a dispositivos electrónicos para la mejora del sistema educativo? Es más, ¿̨por qué aparecen cada vez más profesionales del ámbito de la psicología, la educación, la psiquiatría, desaconsejando el uso generalizado de las tecnologías en casa y/o en las aulas? ${ }^{[10]}$ 
Conviene señalar que, por muy poco populares que puedan resultar estas recomendaciones de abstención, no se mueven por grandes intereses comerciales o políticos, no son pautas ni criterios proporcionados por investigadores pagados por empresas tecnológicas, ni supeditados a subvenciones o consecución de puntos para centros educativos por parte de algún organismo estatal. No obstante, los medios tecnológicos también ofrecen unas ventajas nada desdeñables que conviene exponer a continuación.

El conjunto de los ciudadanos está inmerso en el mundo de las tecnologías, lo queramos o no, incluso si un individuo decide retirarse a un refugio en la montaña. Si se viese en una necesidad, los medios actuales de comunicación actuarían con una rapidez para venir en su auxilio, impensable en la mayor parte del siglo XX. En cierto modo, la tecnología nos ha hecho más fácil la vida: cualquier duda puede ser resuelta con rapidez, las citas pueden concertarse desde los sitios más remotos, la misma información puede ser compartida entre varias personas a la vez, simultáneamente y desde diferentes lugares del globo terráqueo.

Podemos así también destacar algunas ventajas que proporcionan las TIC en las aulas:

1. La agenda digitalizada permite una coordinación más sencilla. Los alumnos disponen de los deberes y exámenes de manera actualizada y continua, lo cual ayudaría especialmente a los más despistados. Los coordinadores académicos, y así los profesores, pueden distribuir la carga lectiva de trabajos y exámenes más equitativamente, pues visualizan lo asignado al escolar por parte de los profesores.

2. Una clase de arte sobre una proyección del cuadro de Las Meninas de Velázquez ofrece la posibilidad de señalar con el puntero detalles que no se visualizaban en la imagen pequeña del libro de texto. Permite también una participación más fluida sobre el análisis de una imagen accesible a todos los alumnos del mismo modo, y con un formato más atractivo que parece, en un principio, centrar más la atención.

3. Se fijan visualmente conceptos aprendidos quizá, en un primer momento, de manera más conceptual.

4. Es posible elaborar proyectos con otros compañeros de manera colaborativa por medio de teleconferencias, y enviar los deberes al profesor.

5. Muchos profesores consiguen motivar mejor al alumnado mediante un uso comedido de las tecnologías en el aula.

Sin embargo, estas mismas ventajas tienen su contrapartida:

1. Cuando el alumnado anota los deberes en una agenda manual, se está dando cuenta del tiempo que va a tener que emplear en los deberes cuando llegue a casa, está aprendiendo a planificar su tiempo. Mientras que en la agenda se le da ya planificado, y el cumplimiento de las tareas se presenta más como deber que como logro, fruto del autodominio y del ejercicio de la inteligencia práctica. Un logro por el cual gozarse (implicando a la voluntad, al entendimiento y a la afectividad). Se habla de chicos y chicas que están desarrollando su autoestima, y para ello es muy importante que experimenten que han sido capaces no porque respondan a lo programado en una máquina, sino porque responden a la llamada de otra persona que espera cada día más de ellos y de ellas. La agenda y el programa no deben sustituir el encuentro.

2. Una clase de arte que se limitase a analizar cuadros visualizados, perdería el contexto y la profundidad de pensamiento que puede dar una clase más conceptual y magistral complementaria. Somos seres comunitarios, crecemos sobre una comunidad científica la cual ya ha hecho muchos descubrimientos, y sobre los cuales podemos llegar más lejos que con el mero sistema autodidacta, aunque este también sea necesario incluirlo para el crecimiento de la conciencia de la propia capacidad y de la significatividad de la propia experiencia. 
3. Si lo conceptual pretendiese extraerse únicamente desde lo visual, se estaría restando lugar a la memoria, a la capacidad de crítica, a la profundidad del pensamiento, a la imaginación y, por tanto, a la creatividad. Estos son elementos propios del ser humano frente a las meras habilidades técnicas del manejo de las tecnologías que tienden a lo contrario cuando se generaliza su uso, como señala L'Écouyer (2015), y explica también Spitzer (2013). Lo visual resta atención a lo conceptual, a menos que sirva para afianzar lo ya comprendido a este nivel, en un segundo momento, o para la apertura de un debate, como puede ser el caso de la escena de una película como ocasión para dar lugar a un debate moral. Sin embargo, la escena puede también afectar a la sensibilidad de tal modo que condicione significativamente el debate sin permitir que se lleve a las últimas consecuencias, especialmente si la escena es previa a la reflexión sobre los conceptos involucrados en la misma.

Por todo ello, retomando la falta de claridad en la mejora del rendimiento académico, no se presenta de manera unívoca la ventaja del uso de las tecnologías en el aula. Retomando los resultados de los estudios de Ophir et al. (2009) sobre la multitarea, en una entrevista a Nass en la National Public Radio (2013), este expresa su preocupación por la falta de atención y efectividad que muestran las personas expuestas a la multitarea tecnológica. Explica cómo esta situación repercute en el ejercicio de la creatividad y la falta de profundidad del pensamiento, que queda habituado a lo irrelevante, frente a otro tipo de actuaciones pedagógicas que procuran un desarrollo integrador del cerebro.

Cualquier programa de ordenador actualmente exige cierta actuación multitarea frente al pensamiento profundo, pausado, medido; exige abrir un documento, pinchar para cambiar de letra, introducir un elemento externo al procesador, etc. Si bien es cierto que no todos los programas ofimáticos y proyectos educativos de colaboración en red exigen el mismo grado de dispersión en la atención, la posibilidad de cambiar, descargar o eliminar programas, está al alcance de los alumnos.

4. Todo lo dicho sobre la importancia del encuentro, y de las relaciones interpersonales vale de todo punto para no reducir la interacción entre los compañeros a un campus virtual. Los iguales son parte fundamental para el crecimiento en el conocimiento de sí mismo, de los propios límites y virtudes. La vivencia de este encuentro con el otro y consigo mismo ante el otro, no debe ser sustituida por un contacto meramente virtual, aunque ayude a salvar distancias cuando sea necesario (Twenge, 2017).

5. En cuanto a la motivación, en un primer momento parece mejorar la disciplina en el aula, aunque no siempre se puede controlar a expensas de qué distracción la está mejorando. Se pone a disposición del educando la herramienta de trabajo y la de ocio. Los alumnos pueden cargar y descargar contenidos y aplicaciones sin que el profesorado se entere, en cuestión de segundos.

Aún si se consiguiese un control sobre esta situación, se estaría socavando la importancia de la propia iniciativa del alumnado la motivación interna. Se estaría exponiendo al alumno a que su atención tuviese que estar siempre motivada desde fuera para mover su voluntad como si se tratase de un ser inanimado o sin la especificidad espiritual propia del ser humano, sino desde principios internos. Feinstein (2006) trata acerca de esta competitividad entre motivación interna y externa. Se trata de promocionar un movimiento del educando desde el propio autodominio, más que desde la inmediatez tecnológica de carácter mayormente abductor, desde la interioridad que muestra la irrepetibilidad del ser humano, y que contribuye a su integración (Oestreicher, 2013; Wrzesniewski, Schwartz, Cong, Kane, Omar, y Kolditz, 2014); aquella que es capaz de trascenderse hacia la otra persona porque se tiene a sí misma como un alguien y no como un mero peón tecnológico. La motivación externa está justificada cuando fortalece la motivación interna en un uso puntual, progresivo, de apoyo; no cuando la suplanta.

En todas estas ventajas que tienen su contrapartida, se corre un riesgo que parece más grave que todas esas contrapartidas, por las consecuencias en la madurez de la persona: el riesgo de la inmediatez. Siendo esta una 
gran ventaja de las tecnologías para conseguir un trabajo más productivo en el ámbito laboral; en la formación de la persona en edad de maduración, se muestra, sin embargo, bastante perniciosa. Ninguno de los objetivos educativos señalados se alcanza inmediatamente: ni el pensamiento profundo basado en conocimientos asentados, ni la capacidad de autodominio, ni la educación del corazón para darle importancia a lo que la tiene en el proceso de personalización, respetando al otro y a sí mismo. El infante y el adolescente, acostumbrado a obtenerlo todo con un clic, también deseará tener acceso a experiencias rápidamente gratificantes en otras vivencias, incluidas las relacionadas con la dimensión sexuada (Twenge, 2017).

Desde la perspectiva de la filosofía personalista de Wojtyla (2011), el hombre se personaliza mediante los actos que le son propios, que presentan "el carácter de una auténtica autodeterminación, en la que se realiza la trascendencia de la persona” (p. 379). Y, se despersonaliza actuando en contra de lo que es propio a sus dinamismos antropológicos. A la luz de la contraposición motivación interna-externa, y de esta definición de la persona desde su acción propia: no resulta igualmente personalizador el encuentro entre educador y educando, que la exposición a la luz azul, incluso cuando esta secunda ese encuentro, no siendo necesaria. Y, si, como veíamos en Stein (1998), un acto predispone para el siguiente: no es indiferente que un niño de educación previa a la universidad, esté configurando su personalidad desde una dedicación temporal ante la pantalla. Máxime cuando esta dedicación está configurando su cerebro de un modo en que no puede hacerlo igual en el adulto $^{[11]}$ (Spitzer, 2013).

\section{LAS TECNOLOGÍAS Y LA REALIZACIÓN DE LA PERSONA}

Ningún acto, que afecte directamente al desarrollo personal, es neutro, por serlo de un fin en sí, con una dignidad. Y, sobre todo, porque la persona busca su realización en la comunión, en el amor interpersonal ${ }^{[12]}$, esto implica sobre todo capacidad de entrega y, por tanto, de autodominio, para ponerse a disposición del nosotros en la elaboración y realización del proyecto común. La exposición tecnológica no es ajena a este criterio: en ella, se generan actos que ocupan el tiempo modificando las tendencias y, con ello, la conducta. El empleo de las TIC puede, así, fortalecer o debilitar la voluntad para ponerla a disposición de otro/Otro. La fortalece, por ejemplo, cuando se emplea de manera puntual para mantener contacto con una persona que, temporalmente, no puede ser tratada en la cercanía. Puede, por tanto, servir a la trascendencia de la persona, pero puede también encerrarla en su mismidad, como apuntábamos en el ejemplo del autismo virtual.

Las tecnologías tienen sus ventajas, pero en una medida en que no afecte al desarrollo de la persona en lo relativo a un fin principal como es la comunión interpersonal que se hace cargo de su ser relacional, y de la respuesta adecuada a la persona en el amor que promueve, y no solo respeta. Su uso será adecuado en la medida en que no sustituya la función principal del y la docente como promotor del educando en todas sus dimensiones personales, y que, por tanto, no ha de quedar reducido a la figura del facilitador/a de información.

La educación, por tanto, tampoco puede quedar reducida a un proceso meramente, o principalmente, autodidacta obviando la realidad comunitaria de la persona. Hay una verdad más grande que la que puede alcanzar la realidad contingente del individuo, o que la propia de la información digitalizada; y que se comunica en la experiencia y el testimonio transmitidos, un testimonio de mucha más fuerza que el tecnológico, en aras a mover la voluntad del educando, y orientarla de manera efectiva y personalista para el fin principal al que tiende el ser humano y que responde de su dignidad: amar y ser amados (Allen, 2014; Scheler, 1998).

En este sentido, es importante el aspecto de la atención: el educando se siente amado en la medida en que se siente atendido, y la exposición a la pantalla limita esta percepción por parte del alumnado, además de reducir su capacidad de atención interpersonal mediante la dispersión e irrelevancia de la realidad a la que da lugar la multitarea (Ophir et al., 2009). Con ello queda reducida la capacidad de sentirse amado, que, a su vez, mueve a amar, a entregarse como respuesta a un amor primero, a focalizarse hacia otro hasta el 
punto de que ese otro forme parte de mí mismo por medio de la sobreactualidad del amor ${ }^{[13]}$. Por medio de la hetero-autoconciencia, doy lugar al otro en la conciencia, tomando decisiones teniendo en cuenta al tú (Nédoncelle, 1996). Se trata de capacitar al educando para que otros se sientan queridos, atendidos por él de manera interpersonal y no solo, principalmente o innecesariamente reductivamente de manera virtual.

Las etapas más críticas en las que se desarrolla el sistema neuropsicológico de la persona, en una orientación personalista, no deben verse condicionadas por una exposición excesiva a los dispositivos electrónicos, cuando esta conlleva que se tienda a tratar con la realidad offline como con la realidad virtual. En un trato interpersonal afectado por el exceso de virtualidad, las otras personas devienen instrumentos facilitadores de información, proporcionan el acceso, y son importantes por ello. Los demás aspectos personales del otro tienden a devenir irrelevantes, por no haber aprendido a prestarles atención, por haber interferido en el proceso de adquisición del dominio necesario sobre la motivación externa.

La exposición continuada a las TIC en el ámbito escolar interfiere, por tanto, con esta capacitación para dar importancia a lo personificante, más allá del mundo virtual. El encuentro se difumina, predispone a considerar al otro más como medio que como fin en sí que ayuda a alcanzar el sentido hondo y profundo de la vida, y disminuye el desarrollo de la propia relacionalidad (Oestreicher, 2013), que encuentra su sentido en el amor como respuesta a las dimensiones de la persona. O bien, consideramos al otro desde una noción vaga de su ser fin en sí, pero no desde una noción que tome en serio la condición existencial real del otro, con la profundidad con la que podría considerarlo fuera de aquel condicionamiento neuropsicológico que conlleva la exposición intensiva a la realidad virtual (Konrath, O’Brien y Hsing, 2011).

El cerebro funciona como un músculo (Spitzer, 2013); si se ejercita, adquiere una habilidad, de lo contrario se incapacita o limita bastante. Es decir, no llega a todo su potencial. Pero somos seres limitados, contingentes, esto implica decidir en qué se van a ejercitar más los hijos e hijas, qué habilidades quieren los padres desarrollar más en ellos, especialmente si unas van en detrimento de otras; cuáles son más necesarias para la vida que queremos vivir. Y, entre la apuesta por una motivación externa que suplante a la interna por una exposición continuada, que tiende a la superficialidad; y la capacidad de abstracción, de atención interpersonal, de memoria que permite aprender de la experiencia y no solo cortar y eliminar, la capacidad de concentración y no solo de abducción; queda justificada la apuesta por la no exposición del alumnado a las pantallas.

El alumnado tecnócrata funciona, al menos en una primera etapa, no molesta; pero no está creciendo en lo que más le personaliza como lo haría sin tanta exposición a la realidad virtual (Nass, 2013; Oestreicher, 2013). Se maneja estupendamente en el mundo tecnológico, pero también es fácilmente absorbido por él, queda en parte anulado como persona, es una rueca más del sistema, eso sí eficaz, pero no sin afectar a su creatividad fuera del mundo digital. Además, es preciso hacer notar que la creatividad es una de las habilidades más importantes a la hora de mantener vivo un amor profundo, y no decaer en la superficialidad de un amor itinerante que en el fondo solo se quiere a sí mismo, y permanece en la reducción del amor al estado de enamoramiento, de lo que se siente de manera inmediata; incapaz de incentivar un proyecto común, como apuntan los estudios de Twenge, Martin et al. (2018). En último término, el que puede crear más allá de lo que existe en el mundo virtual o incluso para el mundo virtual, el que tiene las ideas, es el que revoluciona el mercado, más que el que las sabe presentar en el mercado, que deviene un peón de aquel.

Quizás esto explique que los hijos y las hijas, cuyos padres trabajan en grandes empresas tecnológicas de Sillicon Valley, acudan a centros en los cuales se procura: que no utilicen estos mismos medios ni en casa ni en el aula; que escriban con lápiz y papel, como sucede en el Waldorf of Peninsula. El uso de pantallas queda fuera de las aulas en estos centros educativos y, desde el colegio, se desaconseja en el ámbito familiar (Richtel, 2011).

Tanto Bill Gates como Steve Jobs limitaron el tiempo de pantalla de sus hijos, y Chris Anderson, exdirector de la revista $W$ ired (de temática digital), afirmaba que el uso de la tecnología estaba más cerca del crack, que de los caramelos (Guimón, 2019). Estos directivos son conscientes de lo intuitivo que resulta hoy día aprender a manejar un ordenador, tableta o teléfono inteligente. Esto demuestra en su uso en que pueden ejercer hasta 
dos generaciones anteriores a la actual con cursos de pocas sesiones e incluso sin estos cursos. La elección de estos directivos para la educación de sus hijos queda también justificada por lo obsoleto de los programas actuales. Lo que aprendan hoy de poco les va a servir en 10 años, si de lo que se trata es de no quedarse desfasado en el ámbito tecnológico del que forman parte hoy día prácticamente todas las profesiones.

Sin duda, antes o después, los hijos tienen que tener acceso a estas tecnologías para introducirse en el mercado laboral; pero la educación lo es, en primer lugar, de la persona. La educación ha de estar enfocada al crecimiento de la persona. En cuanto a las habilidades tecnológicas necesarias para la profesionalidad en el mundo actual: un robot podrá llegar a ejecutarlas mejor que un humano, pero nunca podrá reproducir lo propio del ser humano. La persona puede superar al robot en: su capacidad de trascendencia en una creatividad que no se reduce a una combinación de elementos programados; su capacidad de autodominio para esperar el tiempo oportuno de cada persona, para controlarse ante reacciones o circunstancias inesperadas, sin dejarse llevar de la inmediatez; su capacidad de contextualizar la realidad y conducirla a su sentido más humanizante, más allá de la productividad; su capacidad de reducir el dolor de otra persona por medio del amor, con el solo acompañamiento (Twenge, Joiner et al., 2018; Twenge, Martin et al., 2018).

\section{UNA RESPUESTA AL CONOCIMIENTO TECNOLÓgICO DESDE LA TEORÍA PERSONALISTA DEL CONOCIMIENTO DE Burgos (2015).}

En toda esta temática caben muchos matices, pero se vislumbran más riesgos y desventajas, que ventajas en la exposición continuada a los dispositivos electrónicos en niños y niñas de infantil, primaria e incluso secundaria. No así en el aprendizaje del uso de las tecnologías, que puede resultar oportuno, así como de habilidades previas a la ofimática como la mecanografía. Pero, para respetar el desarrollo neuropsicológico e integral de las y los niños y promocionarlo, una cosa es usar la Tablet/iPad o dispositivo electrónico como herramienta de apoyo, puntualmente y de manera progresiva, acompañando el desarrollo de su autonomía y autocontrol; y otra muy distinta, como herramienta de trabajo casi exclusiva. En este sentido, incluso un uso pedagógico con el objetivo de alcanzar el aprendizaje del empleo de las tecnologías, sería recomendable retrasarlo a una edad mínimamente madura del cerebro humano sin interferir en etapas críticas de su desarrollo (Spitzer, 2013), e introducirlo de manera progresiva.

En los estudios señalados sobre la multitarea, se apunta al aumento en la rapidez del procesamiento cerebral que produce la exposición a pantallas, como argumento para introducirlas. Sin embargo, se contraviene este argumento con la profundidad y relevancia de dicho procesamiento que es la que puede competir con el robot. La exposición continuada a la pantalla en edad escolar no solo resulta perniciosa, generando trastornos como el señalado autismo virtual (Oestreicher, 2013); sino que alcanza un aprendizaje obsoleto, lo que aprendan cambiará en un tiempo muy corto. Por lo que habrán perdido el tiempo necesario para desarrollar su cerebro de manera personalizante de sí y de otros. Un aprendizaje tecnológico, además, innecesario: los programas son cada vez más intuitivos.

Una propuesta personalista que quiera justificar el uso de las tecnologías en el aula, en caso de que un centro considere que una exposición moderada favorece el rendimiento académico, tiene que tener en cuenta estos riesgos y afrontarlos desde una teoría del conocimiento que responda de los dinamismos de la persona. En este sentido, Burgos (2015), sin pretender dar respuesta a esta temática, aporta un criterio válido para el uso de las tecnologías como herramienta puntual de apoyo desde la realidad virtual cuando afirma, en referencia a la fenomenología realista:

no apostar por un realismo decidido no es una posición epistemológica neutra, tiene consecuencias, y son negativas. La principal es una pérdida de realidad del objeto fenomenológico que queda reducido al carácter de fenómeno, un planteamiento que acaba pasando factura ya que acaba empobreciendo -y a veces deformando- la realidad [...] Desde la experiencia integral [la esencia]... debe estar siempre pegado a la experiencia ya que la comprensión no trasciende totalmente a la experiencia (pp. 115, 120). 
Es decir, la inmersión en la realidad virtual puede ocupar el lugar de la epoché fenomenológica: presentar visiblemente la esencia del conocimiento que estamos tratando de mostrar al alumno. Pero, desde la realidad virtual, e incluso antes de la inmersión en ella, el contenido a aprender ha de volver a ser comprendido desde la realidad personal concreta, permitiendo en el alumnado su interiorización por medio de la experiencia propia y ajena (la del profesorado). De manera que dicho contenido pueda ser memorizado y aprendido adecuadamente, gracias al concurso de una subjetividad personal espacio-temporal en interacción con el profesorado que corrobora lo experimentado, con el papel, con el tiempo, con la irreversibilidad propia de lo real, y menos de lo virtual, que ha de ser aprehendida por la memoria, porque no se puede deshacer, y quizá conviene no repetir o reforzar.

La implantación de las TIC en los centros educativos, más que en las aulas si no se quiere derivar en un uso intensivo de las mismas, ha de ser revisable, crítica y limitada: ajustarse al proceso personalizador del educando. Si se quiere acompañar el desarrollo de la persona respetando sus ritmos, hay que evitar imponer un modelo educativo tecnológico por motivaciones ajenas a los dinamismos internos de la persona (Burgos, 2003; Stein, 1998).

\section{RAZONES DE LA IMPLANTACión DE LAS TIC EN EL AULA}

Los estudios que dan lugar a afirmaciones como las de la OCDE (2015), descartan que el uso de la tecnología como medio habitual de aprendizaje, aumente el rendimiento académico de los alumnos. También hay estudios que demuestran lo pernicioso de su uso prolongado en el tiempo, como los recogidos por Spitzer (2013) o Twenge, Joiner et al. (2018), Oestreicher (2013), o Dunkley (2015). Asimismo, hay países como Suecia, que retiran los dispositivos electrónicos de las aulas; o Francia, que prohíbe el uso de móviles en el colegio, también en los recreos, y que achaca a cualquier tipo de pantalla digital la disminución de la concentración en el alumnado hasta los 15 años (DJO, 13 diciembre, 2017).

¿Por qué entonces el auge en países como España del uso de las tecnologías en el aula? L’Ecouyer (2015) señala algunas de las razones para la implantación de las TIC.

En ocasiones son los padres quienes solicitan esta introducción por miedo a que sus hijos no puedan entrar adecuadamente en el mercado laboral. Es decir, no porque estén preocupados por lo que puedan aprender a nivel humano o incluso de conocimientos, sino únicamente con el fin de conseguir una habilidad técnica. Ahora bien, teniendo en cuenta lo obsoleto de estos programas, o lo intuitivo de los mismos, sería suficiente un aprendizaje puntual para paliar este escollo en el mundo global en el que nos encontramos.

Bastarían un par de horas a la semana de informática, mecanografía u ofimática, si se quiere insistir en la cuestión, obviando lo intuitivo de su aprendizaje en la actualidad; o una implantación progresiva; o se podría retomar la inmersión en el lenguaje tecnológico a los últimos cursos de la educación secundaria. Así lo entienden y aplican colegios tan diferentes en ideario como el Mater Salvatoris, el Colegio Internacional Kolbe, el colegio Estudio, los centros Waldorf, o los colegios Juan Pablo II. Cada uno con una implantación distinta, pero crítica y limitada.

Otra de las razones que se esgrime es que los profesores consiguen motivar mejor a los alumnos mediante estos medios, pero ¿consiguen centrarlos mejor? ¿por cuánto tiempo consiguen motivarlos mejor y de igual manera? ¿consiguen que desarrollen mejor un pensamiento creativo, crítico, profundo? ¿el hecho de que estén acostumbrados al mundo virtual fuera del aula es un criterio suficiente para mantenerlos en esa realidad dentro del aula? Una costumbre o novedad no es buena o inamovible simplemente por ser costumbre o novedad, no personaliza por el mero hecho de ser costumbre o novedad. Los estudios señalados en este ensayo sobre la multitarea apuntan a lo contrario respecto de la relación entre la motivación y la profundidad del pensamiento.

Sin duda, en alumnos con poca motivación para cursar estudios superiores, es posible que se produzca una motivación externa que supla la falta de motivación interna, pero ¿el profesorado ha de facilitar la sustitución 
de la motivación interna por la externa, o más bien ha de usar la externa para llevar a la interna y consolidarla? La perspectiva personalista integral aboga por la segunda opción. En palabras del pensador personalista Guardini (2002): "La correspondiente misión del educador es permitir que el niño desarrolle su propio modo de ser y animarle incluso a que se acostumbre a obrar por propia iniciativa” (p. 41).

Un director de colegio puede verse motivado a introducir iPads o tabletas como herramienta de estudio más que de apoyo, debido a: la demanda de los padres, la conveniencia de los profesores o un primer entusiasmo del infante, o del adolescente por esta introducción, ya que se mueve en el mundo virtual con más facilidad que en el conceptual. Un entusiasmo que bien puede derivar en una posible adicción, insomnio, falta de concentración, la costumbre que lleva al hastío de compartir todo, también el trabajo escolar, por medio de relaciones superficiales, sin interioridad, sin encuentro (Twenge, Joiner et al., 2018; Twenge, Martin et al., 2018).

En la visión personalista expuesta en este ensayo, la educación es concebida como una misión en la que el objetivo es un fin en sí, la persona (Stein 1998), y no la consecución de un producto con habilidades especiales con el que se puede experimentar sin la máxima cautela ante criterios educativos de alcance cuestionable. A la luz de una pedagogía integral de corte personalista, las razones aludidas para la implantación de las TIC no obedecen a criterios puramente pedagógicos: ¿ha de someterse el centro educativo a la ley de la oferta y la demanda para la aplicación de las TIC? Un centro educativo debería ser más que una empresa, más que un lugar para la reivindicación de medios.

El objetivo de la educación bajo el prisma del personalismo integral, es el crecimiento de la persona en tanto que tal (Guardini, 2002), por medio, sobre todo, del encuentro (Scheler, 2001). Es por ello, que la pantalla, en una visión personalista del tema que nos ocupa, no debería sustituir al profesor si quiere motivar ese crecimiento desde dentro. Tampoco debería sustituir el encuentro con el alumnado por una retroalimentación telemática, si quiere mantener también el crecimiento personal del profesorado como vocación, aquella que le lleva también a él a una plenitud no sustituible por la figura del facilitador/a de información ${ }^{[14]}$

El deseo legítimo del padre y/o de la madre de capacitar a los hijos; el recurso a la motivación externa por parte del profesorado; el marketing del director de un colegio; el entusiasmo previo del alumnado, no son razones incuestionables para llamar educación a una exposición continuada a los dispositivos electrónicos. La educación busca un objetivo para el cual son necesarios criterios más trascendentes, centrados en la persona desde el encuentro si quiere contribuir a un proceso personalizador de realización del alumnado en sus distintos dinamismos. Si bien, se mantiene una diferencia entre el uso de las tecnologías como herramienta de apoyo puntual, y el uso de las tecnologías como herramienta de trabajo fundamental. Esta diferencia permite a una visión personalista no excluir el uso de las tecnologías como herramienta de apoyo en el ámbito educativo, pero tampoco necesariamente lo exigiría por su carácter de obsoleto, intuitivo, e incluso problemático a la hora de alcanzar el desarrollo pleno de otras habilidades más humanamente fundamentales, más específicamente personales.

Como ya se señaló que afirmaba la AAP (2016), en caso de que los padres así lo deseen, pueden introducir las TIC de manera puntual sin exponer a sus hijos en exceso a los trastornos indicados. Ahora bien, en palabras de Pierre Laurent (citado por Guimón, 2019), padre de tres hijos, ingeniero informático que trabajó en Microsoft, Intel y diversas startups, y que ahora preside el patronato del colegio Waldorf of Peninsula:

La creatividad es algo esencialmente humano. Si le pones una pantalla a un niño pequeño limitas sus habilidades motoras, su tendencia a expandirse, su capacidad de concentración.... Tendremos las respuestas [sobre las consecuencias de la implantación de las TIC en el aula] en 15 años, cuando estos niños sean adultos. ¿Pero queremos asumir el riesgo? (párr. 3) 


\section{Conclusiones}

El objetivo de este trabajo era facilitar criterios antropológicos para la evaluación del impacto del uso de las TIC en el crecimiento personalizador del educando. Se ha acudido al personalismo integral, por ofrecer unos fines muy definidos de la educación en Stein (1998), sobre la base de una antropología que valora los dinamismos de la persona de manera integral (también queda patente en Burgos, 2003; Wojtyla, 2011).

Los estudios presentados en el primer apartado indican la necesidad de cautela en el empleo de las TIC, por estar su abuso asociado a disminución del rendimiento académico, así como de otras habilidades no tecnológicas como las matemáticas y la escritura, y a la afectación de factores de la vida cotidiana como el sueño, la actividad física, y las relaciones interpersonales.

En los siguientes cuatro apartados, se aborda la concepción de la persona en el personalismo integral: como una persona contingente, con una fuerza limitada en el empleo de sus facultades. Por lo que se propone un sistema educativo basado en la orientación, acompañamiento, guía del y la docente al educando; como sistema más adecuado al crecimiento personal, que el meramente autodidacta. La importancia del y la docente, para este crecimiento, queda antropológicamente fundamentada por la importancia del desarrollo de la interioridad por medio del encuentro que despierta al tú mediante el diálogo con el yo, y mediante el enriquecimiento que supone una relación no meramente horizontal y homogénea: la del y la docente como prototipo.

El y la docente, así entendido, interpela el dinamismo del educando, activando la motivación interna, por encima de la externa, más propia de los medios tecnológicos. En este sentido, se considera que, para involucrar a la afectividad en el proceso (como motora de la voluntad, y, de este modo, de la motivación intrínseca): a) el tiempo debe distribuirse en el aula de manera que pueda darse el encuentro; y b) la continuada exposición a las pantallas, interfiere negativamente en este encuentro. Un encuentro en el que se ofrece al educando la posibilidad de desarrollar lo más propio del ser humano: su autoconciencia, autodominio y autotrascendencia. Se ha mostrado también cómo, dada la unicidad de la persona, y de sus experiencias; una educación más basada en el encuentro, contribuye a un bienestar superior al que ofrece la exposición continuada a las TIC; señalando los aspectos negativos asociados a dicha exposición en estudios concretos.

En el sexto apartado, se han valorado las ventajas y desventajas de empleos puntuales de las TIC en el aula. Se destaca aquí que el empleo no es neutro o bueno solo por ser moderado, sino que han de tenerse en cuenta otras desventajas de dicho empleo, y un orden de preferencias en el desarrollo de otras habilidades, que de sentido a su empleo. Se señala, especialmente, el riesgo de la inmediatez, como factor propiciado por el empleo de las TIC, y pernicioso en el proceso de maduración del educando, aunque pueda resultar muy positivo en el ámbito laboral.

El séptimo apartado, aborda un término muy característico del personalismo integral: el destino, o realización de la persona; focalizándolo en la comunión, o amor interpersonal, capaz de un proyecto común. A tales efectos, el encuentro contribuye más, que la exposición a pantallas, al desarrollo de la autoconciencia, el autodominio, y la autotrascendencia; así como a la capacidad de entrega del educando y, por ende, a su realización en sentido personalista. Por lo que, retomando también los anteriores criterios personalistas, entre ellos destacando la contingencia humana, queda justificada la decisión de no exponer al alumnado a las pantallas. Se resalta lo intuitivo y obsoleto del aprendizaje del lenguaje tecnológico en la formación correspondiente al período escolar, previa a la formación superior; y la importancia de desarrollar otras habilidades no tecnológicas con las que no pueda competir un robot en el mercado laboral.

En el octavo apartado, se ofrece el criterio gnoseológico personalista de Burgos (2015), para dilucidar acerca de la pertinencia o no del empleo de las TIC: la experiencia virtual debe poder permitir al educando volver siempre a la experiencia integral. El empleo de las TIC ha de ser, en este sentido: revisable, crítico, y limitado.

En el noveno apartado, se valoran las razones indicadas por L'Écouyer para la importancia de las TIC en los centros educativos, según los criterios expuestos en los anteriores apartados. Desde estos criterios, se indica 
que ha de prevalecer la motivación intrínseca del educando sobre la motivación del centro, del profesorado, de las madres y los padres, o de la extrínseca del propio educando; y se concluye que esta motivación extrínseca es más propia de las TIC.

\section{ReFERENCIAS}

Academia Americana de Pediatría [AAP] (2016). Media and Young Minds: Council on Communications and Pediatrics. Pediatrics, 138(5), 1-8. Recuperado de https://pediatrics.aappublications.org/content/138/5/e201 62591

Agencia EFE, (2 agosto del 2018). Dispositivos electrónicos afectan biodesarrollo a entre 10 y 15\% de niños. Sección Edición América. Recuperado de https://bit.ly/33wNI8f

Allen, R. T. (2014) Ethics as Scales of Forms. Newcastle: Cambridge Scholars.

Beland, L.P. y Murphy, R. (2016). Communication: Technology, Distraction \& Student Performance. Labour Economics, 41, 61-76. Recuperado de https://www.sciencedirect.com/science/article/abs/pii/S092753711630 0136

Burgos, J. M. (2003). Antropología: una guia para la existencia. Madrid: Palabra.

Burgos, J. M. (2012). Introducción al personalismo. Madrid: Palabra.

Burgos, J. M. (2015). La experiencia integral. Madrid: Palabra.

Burgos, J. M. (2019). Wojtyla's Personalism as Integral Personalism. Quaestione disputate, 9 (2), 91-111.

Carr, N. (2011). Superficiales: ¿qué está haciendo Internet con nuestras mentes? Madrid: Taurus.

Crosby, J. F. (2007). La interioridad de la persona humana. Madrid: Encuentro.

Dunkley, V. (2015). Reset Your Child's Brain: A Four-Week Plan to End Meltdowns, Raise Grades, and Boost Social Skills by Reversing the Effects of Electronic Screen-Time. Novato, CA: New World Library.

Enkvist, I. (2012). La buena y la mala educación. Madrid: Encuentro.

Feinstein, S. (2006). The Praeger handbook of learning and the brain. Westport, CT.: Praeger.

DJO. (13 diciembre, 2017). Francia prohíbe el uso de móviles en los colegios... hasta en el recreo. El mundo. Recuperado de https://bit.ly/2Gc64Cv

Fuchs, T. y Wössmann, L. (2005). Computers and Student Learning: Bivariate and Multivariate Evidence on the Availability and Use of Computers at Home and at School. Ifo Working Paper, 8, 1-28.

Guardini, R. (2002). Las etapas de la vida. Madrid: Palabra.

Guimón, P. (2019, 24 marzo). Los gurús digitales crían a sus hijos sin pantallas. El País. Recuperado de https://bit. ly/2KOKXqv

Heffler, K.F. y Oestreicher, L.M. (2016) Causation Model of Autism: Audiovisual Brain Specialization in Infancy Competes with Social Brain.Medical hypotheses, 91, 114-122. doi: https://doi.org/10.1016/j.mehy.2015.06.019

Konrath, S. H., O’Brien, E. H. y Hsing, C. (2011). Changes in Dispositional Empathy in American College Students Over Time: a Meta-Analysis. Personality and Social Psychology Review, 15(2), 180-198. doi: https://doi.org/10 $.1177 / 1088868310377395$

L'Écuyer, C. (2015). Educar en la realidad. Barcelona: Plataforma Editorial.

Madigan, S., Browne, D., Racine, N., Mori, C. y Tough, S. (2019). Association Between Screen Time and Children's Performance on a Developmental Screening Test.JAMA Pediatrics, 173 (3), 244-250. doi: https://doi.org/10 $.1001 /$ jamapediatrics.2018.5056

Nass, C. (2013, 10 mayo). The Myth of Multitasking. National Public Radio. Recuperado de https://www.npr.org/2 013/05/10/182861382/the-myth-of-multitasking

Nédoncelle, M. (1996). La reciprocidad de las conciencias. Madrid: Esprit.

Ophir, E., Nass, C. y Wagner, A. D. (2009). Cognitive Control in Media Multitaskers. PNAS, 106 (37), 15583-15587. doi: https://doi.org/10.1073/pnas.0903620106 
Oestreicher, L. (2013) The Pied Pipers of Autism-How TV, Video, and Toys Cause ASD. Scotts Valley: CreateSpace Independent Publishing Platform.

Organización para la Cooperación y el Desarrollo Económicos [OCDE] (2015) Students, Computers and Learning: Making the Connection. (pp.15-26). Luxemburgo: OECD Publishing. doi: https://dx.doi.org/10.1787/97892 64239555-en

Pliego, F. (2013). Tipos de familia y bienestar de niños y adultos. México: UNAM-Instituto de Investigaciones Sociales.

Richtel, M. (2011, 22 octubre). A Silicon Valley School That Doesn't Compute. The New York Times. Recuperado de https://nyti.ms/2keileD

Scheler, M. (1998). Ordo amoris. Madrid: Caparrós.

Scheler, M. (2001). Ética. Madrid: Caparrós.

Spitzer, M. (2013). Demencia digital. Barcelona: Ediciones B.

Stein, E. (1998). La estructura de la persona humana. Madrid: BAC.

Szalay, M. (2016). La transformación de la persona: fantasía o imaginación. Quién, 4, 67-87.

Twenge, J.M. (2017). iGen: Why Today's Super-Connected Kids Are Growing Up Less Rebellious, More Tolerant, Less Happy, and Completely Unprepared for Adulthood, and What That Means for the Rest of Us. New York: Atria Books.

Twenge, J.M., Joiner, T.E., Rogers, M.L. y Martin G.N. (2018). Increases in Depressive Symptoms, Suicide-Related Outcomes, and Suicide Rates Among U.S. Adolescents After 2010 and Links to Increased New Media Screen Time. Clinical Psychological Science, 6 (1), 3-17. doi: https://doi.org/10.1177/2167702617723376

Twenge, J.M., Martin, G.N. y Campbell, W.K. (2018). Decreases in Psychological Well-Being Among American Adolescents After 2012 and Links to Screen Time During the Rise of Smartphone Technology. Emotion, 18 (6), 765-780. doi: https://doi.org/10.1037/emo0000403

Vargas, T. y Polaino, A. (1996) La familia del deficiente mental: un estudio del apego afectivo. Madrid: Pirámide.

Vera, R. (2011) Ontología y gnoseología del yo personal. Madrid: FUE.

Von Hildebrand, D. (1983). Ética. Madrid: Encuentro.

Von Hildebrand, D. (1996). Las formas espirituales de la afectividad. Revista Facultad de Filosofía de la Universidad Complutense, 19, 9-28.

Von Hildebrand, D. (1997). El corazón: un análisis de la afectividad humana y divina. Madrid: Palabra.

Von Hildebrand, D. (1998). La esencia del amor. Pamplona: EUNSA.

Wojtyla, K. (2009). El don del amor. Madrid: Palabra.

Wojtyla, K. (2011). Persona y acción. Madrid: Palabra.

Wrzesniewski, A., Schwartz, B., Cong, X., Kane, M., Omar, A. y Kolditz, T. (2014). Multiple Types of Motives don't Multiply the Motivation of West Point Cadets. PNAS, 111 (30), doi: https://doi.org/10.1073/pnas.1405298 111

\section{Notas}

[1]Para una visión general del concepto de personalismo y de la ubicación de su acepción como integral, y de los autores que lo componen, véase Burgos (2012). El autor de este libro modificó la acepción de Personalismo Ontológico Moderno por la de Personalismo Integral a partir de Burgos (2019).

[2] Aunque resulta conveniente indicar también uno de los más recientes publicado en JAMA Pediatrics por Madigan, Browne, Racine, Mori y Tough (2019), al cual se recurrirá a lo largo del ensayo.

[3] Así, encontramos en el personalista Crosby (2007), la siguiente afirmación:

No me poseo primero y después participo en la subjetividad de otros, sino que la autoposesión puede realizarse plenamente sólo como un reflejo de esa participación. Y no sólo eso, sino que hay una verdad sobre mí mismo de la que otros, precisamente en cuanto otros, son los custodios naturales, como vimos. Otros son los que median entre yo y yo mismo de las más diversas formas. Pero al 
estar así ordenada a la relación interpersonal, mi condición de persona se ordena a una pluralidad de personas -lo interpersonal exige al menos dos personas. Pero esto quiere decir, que mi condición de persona está intrínsecamente vinculada a la finitud.... La persona está como un yo frente a un tú. (p. 352)

[4] Adviértase que puede resultar necesario en circunstancias especiales de distancias insalvables por los contextos que también identifican a la persona en tanto que se entiende desde ellos.

[5] Adviértase el uso antropológico del adjetivo espiritual que hace referencia a las dimensiones volitivo-intelectivas del ser humano. No se trata aquí, por tanto, de un uso propiamente religioso del término.

[6] Estructura con la que se coincide mayormente como queda descrito en el libro de Vera (2011). Por otro lado, desde el punto de vista psicológico, son interesantes también las conclusiones de Vargas y Polaino (1996) en relación a la importancia del cuidador y su relación con el infante.

[7] Un porcentaje significativo que no excluye la posibilidad de problemas en aquellos que no los padecen de manera patológica, pero que quizás podrían tener una mayor calidad de vida y desarrollo personalizador de sus facultades más humanas en ausencia de esta exposición reiterada. Para esta valoración, resulta de especial interés la publicación de Oestreicher (2013).

[8] Esta temática es también abordada por Heffler y Oestreicher (2016), y Madigan et al. (2019); y las afirmaciones del Dr. Eduardo Barragán con ocasión del Primer Congreso de Neuropediatría en Méjico según la nota de la Agencia EFE (2018), sobre cómo están relacionados los dispositivos electrónicos con trastornos de biodesarrollo, entre los cuales menciona el Trastorno por Déficit de Atención e Hiperactividad (TDAH).

[9]Este ensayo también se ve avalado por los estudios de Beland y Murphy (2016), que incluso asocian la ausencia de dispositivos electrónicos en el aula con un aumento del $6 \%$ en el rendimiento académico.

[10] Nacho Calderón, director del Instituto de Neuropsicología y Psicopedagogía Aplicadas (INPA) en Madrid; la investigadora Catherine L'Écuyer; María José Calvo, doctora y orientadora familiar; el psiquiatra Luis Rojas Marcos, son algunos de los nombres junto con los ya enumerados en los estudios estadísticos reseñados en este ensayo.

[11] Si bien, también hay advertencias acerca de cómo en el cerebro del adulto la dedicación de tiempo a la pantalla produce modificaciones considerables (Carr, 2011). Sin embargo, en este ensayo se ha optado por delimitar las repercusiones a la población escolarizada.

[12] Este es un posicionamiento propio de personalistas como Burgos (2003) o Wojtyla (2009); y queda refrendado por estadísticas sociológicas acerca de la importancia de la familia como las que presenta Pliego (2013).

[13]Término propio de von Hildebrand (1998) que hace referencia a la capacidad de que el amado esté presente de manera constante en el trasfondo de mis decisiones, pensamientos y sentimientos, no de manera consciente, pero tampoco propiamente inconsciente.

[14] He de remarcar que se habla en este ensayo siempre de la educación anterior a la formación superior, no analizo la repercusión en los jóvenes de la reducción de su instrucción al mundo online. Quiero así recalcar que los criterios que se apliquen en la Universidad para el uso de las TIC no pueden ser los mismos criterios educativos que se apliquen en un niño o niña en pleno desarrollo neuropsicológico. Somos limitados, y, en nuestra contingencia, estamos sujetos al desarrollo paulatino que se asienta, pero no se enquista necesariamente, sino que puede seguir viéndose afectado por la reducción del conocimiento y la experiencia de la persona al mundo virtual, pero no en igual medida cuando la persona se encuentra en etapas de desarrollo críticas.

\section{BY-NC-ND}

